\title{
Effectiveness Evaluation Method of Anti-Radiation Missile against Active Decoy
}

\author{
Junyao Tanga ${ }^{\mathrm{a}}$, Fei Cao ${ }^{\mathrm{b}}$, and Sijia $\mathrm{Li}^{\mathrm{c}}$ \\ Xi'an Research Institute of High Technology, Xi'an, China \\ a514492278@qq.com, bcaofeimm@163.com, c13259798735@163.com
}

Keywords: Anti-Radiation Missile, Anti Active Decoy, Damage Probability, Effect Evaluation Method.

\begin{abstract}
In the problem of anti-radiation missile against active decoy, whether the ARM can effectively kill the target radiation source and bait is an important index for evaluating the operational effectiveness of the missile. Aiming at this problem, this paper proposes a method to evaluate the effect of ARM against active decoy. Based on the calculation of ARM's ability to resist the decoy, the paper proposes a method to evaluate the decoy resistance based on the key components of the hitting radar. The method has the advantages of scientific and reliability.
\end{abstract}

\section{Introduction}

Anti-Radar Missile (Anti-Radar Missile), also known as anti-radar missiles, is a special attack radar and other radiation source of fast, accurate tactical missile. It guides the enemy radar as a guide, tracks and destroys the radiation source. In the modern war, such as the Gulf War, the Kosovo war, the Vietnam War in the hands and feet, has become a veritable "radar killer".In order to resist the threat of ARM to the entire information war, a series of defensive measures have appeared. Active decoy technology has become one of the most effective anti ARM methods, which greatly affects the missile hit accuracy. In order to improve the development of ARM technology, it is necessary to study the method of evaluating the effect of decoy.

\section{Effect Evaluation Method}

The effectiveness of anti radiation missile anti active decoy, anti decoy effect, not simply to ARM hit the target and the miss two logic to judge. In order to obtain the operational effectiveness, the damage effect of the radar and bait should be evaluated.The western countries on the typical target damage specific classification, such as the flight is divided into: Class KK (the first time the complete destruction of the target (five minutes), Class Class A (five minutes to eliminate the target), Class B (two hours to eliminate targets), $\mathrm{C}$ (the main task that the enemy wants to complete is blocked, it can only be used for secondary purposes), Class D (resulting in additional repair and repair of the enemy), Class E (resulting in the enemy is difficult to complete the task) and other damage levels. Armored vehicles are divided into: Class K (completely destroyed), Class F (fire loss), Class M (caused by the inability to maneuver) and other damage levels. Therefore, the evaluation of anti decoy effect can refer to the above damage classification level, and estimate the damage probability of ARM warhead to the target. In the case of non confrontation environment and system failure, the damage probability of single ARM to a single radiation source is called ARM warhead damage probability [1].

\section{The Calculation of ARM Anti Decoy Damage Probability}

The damage probability is one of the most important indexes to evaluate the performance of ARM against active decoy. The damage probability includes the damage probability of the protected radar and the damage probability of the $i$ active decoy. The traditional evaluation method is based on the distance from the point source of the ARM to the source of the ARM, which can be used to deduce the probability of the radar seeker[2].Let ARM fight into the category of tribal decoy array, if the target and the radar active decoy is effective damage, the distance between the requirements (1): 


$$
\left|O_{i} O^{\prime \prime}\right| \leq R_{d}+R_{r}
$$

$R_{d}$ is the ARM warhead damage radius, $R_{r}$ is a safe point for radiation point source. $\left|O_{i} O^{\prime \prime}\right|$ is the distance between the final placement of the ARM warhead and the i-th point source. Assuming the placement of $O^{\prime \prime}$ coordinates is $\left(x^{\prime \prime}, y^{\prime \prime}, z^{\prime \prime}\right)$, The coordinates of the $\mathrm{i}$-th point sources is $\left(x_{i}, y_{i}, z_{i}\right)$.So then

$$
\left|O_{i} O^{\prime \prime}\right|=\sqrt{\left(x_{i}-x^{\prime \prime}\right)^{2}+\left(y_{i}-y^{\prime \prime}\right)^{2}+\left(z_{i}-z^{\prime \prime}\right)^{2}}
$$

There are N ARM drop points on the location of the source-induced system, If the ARM placement position and the $\mathrm{i}$-th point source do not meet the above inequality have $n_{i}$ points, then the $\mathrm{i}$-th point source damage probability is:

$$
P_{i}=n_{i} / N
$$

According to the probability of knowledge available, the probability of damage to the entire active induced system is:

$$
P_{\text {总 }}=\sum_{i=0}^{n} P_{i}
$$

Based on the above description of the probability of damage, this article will ARM hit the i-th radiation source probability is defined as:

$$
P_{i}= \begin{cases}0 & \left|O_{i} O^{\prime \prime}\right|>R_{d}+R_{r} \\ \frac{\left(R_{d}+R_{r}\right)^{2}}{\left|O_{i} O^{\prime \prime}\right|^{2}} & \left|O_{i} O^{\prime \prime}\right| \leq R_{d}+R_{r}\end{cases}
$$

There are $\mathrm{N}$ ARM drop points on the location of the source-induced system, and the average of $\mathrm{N}$ points is:

$$
\bar{P}_{i}=\frac{1}{N} \sum_{j=1}^{N} P_{i}(j)
$$

$P_{i}(j)$ represents the probability of damage to the i-th point source. The probability that the i-th radiation source and the entire active ejection system are destroyed are:

$$
P_{i}=\frac{1}{N} \sum_{j=1}^{N} P_{i}(j) P=\frac{1}{N} \sum_{i=0}^{n} \sum_{j=1}^{N} P_{i}(j)
$$

\section{Evaluation Method of Anti - seizure based on Hit down of Radar Key Components}

Damage probability depends on the vulnerability of the radiation source, ARM warhead fuze parameters, seeker guidance parameters, ARM with the radiation source of the encounter conditions.In the same coordinate system, the coordinate distribution density $\varphi(X, Y, Z)$ of the drop point $(X, Y, Z)$ can be called the error rule.If the ARM warhead at the point of explosion, there will be a corresponding with the corresponding radiation source damage probability, called the coordinate damage law [3].Coordinate damage laws are closely related to ARM warhead efficiency and radiation source vulnerability. Because the position of ARM drop point has a certain randomness, the probability of anti-perturbation is a reasonable probability of damage, and the conditional distribution density is related to ARM guidance error and fuze start law. According to the probability formula, the probability of successful single-shot is:

$$
P=\iint_{-\infty}^{\infty} \int G(X, Y, Z) \varphi(X, Y, Z) d X d Y d Z
$$

ARM warheads are usually fragmented. The warhead uses the high-speed collision of the fragments to effectively dampen the radiation source such as radar. Thus, the probability of damage is related to the number of fragments and the motion parameters of the fragment at the point of impact 
of the radar radiation source. Fragmentation Warhead Coordinate Damage Probability Description Method is characterized by the probability of the damage of the coordinates written as a single fragment damage probability function. The damage probability of the fragment is related to the motion parameters of the radar radiation source, the vulnerability of the radiation source of the radar and the distance from the radiation source to the radar radiation source. The number of fragments of the radar radiation source is related to the coordinates of the landing point and the critical size and distribution. According to the characteristics of radar radiation source and the power of warhead, the relationship between damage probability and drop point coordinates is established by using geometric relation and vector synthesis.

The application of analytical calculation method to determine the ARM attack efficiency index is difficult, but can be simplified, the analytical solution obtained, the biggest advantage of this solution is more intuitive, has great reference value for understanding ARM's attack efficiency.

The fragment of ARM warhead is composed of metal shell. In order to meet the requirements of a certain shape, size and weight of fragments, the prefabricated or semi prefabricated shell structure is adopted in the design of the shell, so as to obtain the fragments with relatively regular shape, weight and quantity. For this kind of shell structure, some parts of the shell metal explosion after being crushed into a small powder particles, the radar radiation source is not effective, the weight loss of fragments in general about 10\% 15\%.

The warhead is detonated by a laser proximity fuze or a trigger fuze, and the velocity of ARM is relative to the vector of the ARM velocity and the initial velocity of the fragment. Even if all the fragments of the same growth rate, because the moving direction of the fragment, the synthesized value of initial velocity of fragments is also different. The initial velocity of the fragment relative to the ARM projectile at the tip of the warhead is one of the research aspects [4].

Calculate the initial velocity of the fragments when abroad often use Gurney equation, the premise is: product expansion speed along the direction of the linear distance distribution; ARM warhead fuze signal instantaneous detonation; loaded warhead in explosive energy in internal energy conversion for all products and product shell energy. The law of conservation of energy:

$$
V_{0}=D_{e} \sqrt{\frac{\beta}{5(\beta+2)}}
$$

Among them, $D_{e}$ is the instantaneous detonation velocity of explosive; $\beta$ is the mass ratio of explosive loaded to the shell.

Warhead detonated after a damage zone in space, with the effective damage radius, direction angle and scattering angle to describe the damage zone size and shape. Radius of damage with vulnerability of radar radiation source is not a different direction angle and scattering angle of flight varies with ARM. Therefore, the research on the size and shape of the damage area should be combined with the characteristics of radar emitter, warhead and the state of the encounter. The first analysis of the static scattering feature of the fragments, and then study the dynamic scattering characteristics.

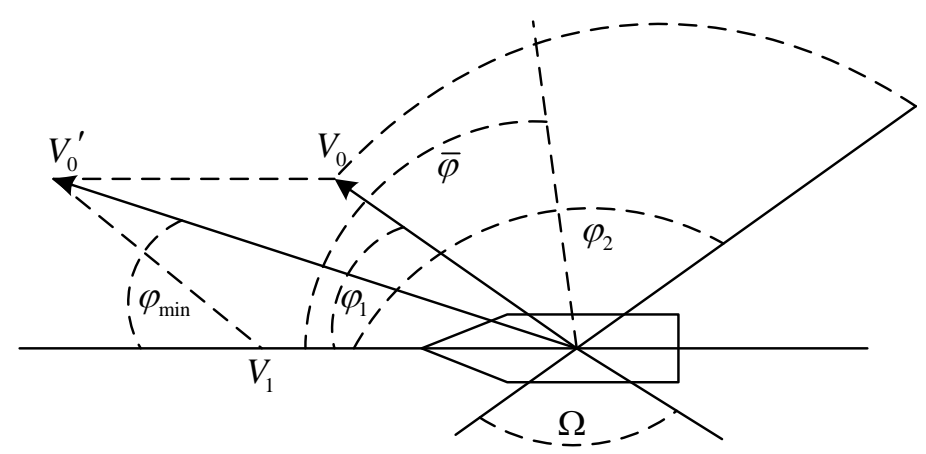

Fig.1 Schematic diagram of the fragment

Warhead shape, structure and loading of explosives and detonating manner decided static scattering characteristic fragment. And the scattering characteristics are defined by the parameters such as the scattering angle $\Omega$, the mean direction angle $\bar{\varphi}$ and the variance $\sigma_{\varphi}$ of the distribution 
density of the fragments. Flying angle $\Omega$ in the axial section, the cone angle centered on the center of gravity of the charge, including $90 \%$ effective fragments:

$$
\Omega=\frac{40 \sqrt{Q_{V}}}{D_{e} \sqrt{\frac{1}{K_{a}}-\frac{1}{2}}}\left(\cos \varphi_{2}-\cos \varphi_{1}\right)
$$

$Q_{V}$ is the explosion to produce heat; $K_{a}=$ explosive weight $/$ (explosive weight + shell metal weight); $\varphi_{1}, \varphi_{2}$ is the angle between the shell surface and the wave direction when the detonation wave reaches the front and back limits. The average direction angle $\bar{\varphi}$ refers to the angle between the axis of the axle and the center of the distribution of the density of the core. For the case where the fragments are completely symmetrical along the distribution center, the geometric bisector of the flying angle is both the centerline. Fragment distribution function in the form of a normal distribution, from the above we can see that $90 \%$ of the number of fragments in the scattering angle is valid, then the distribution of variance $\sigma_{\varphi}$ and the relationship between the scattering angle:

$$
\sigma_{\varphi}=\frac{1}{3.3} \Omega
$$

The dynamic scattering velocity $V_{0 g}$ is the vector sum of the velocity $V_{m}$ and the static scattering velocity $\mathrm{X}$, and the mathematical expectation of the scattering direction and the dynamic scattering velocity is:

$$
\begin{aligned}
& \overline{\varphi^{\prime}}=\arctan \frac{\overline{V_{0}} \sin \overline{\varphi_{c}}}{\overline{V_{0}} \sin \overline{\varphi_{c}}+V_{m}} \\
& \overline{V_{0 g}}=\sqrt{{\overline{V_{0}}}^{2}+V_{m}{ }^{2}+2 \overline{V_{0}} V_{m} \cos \overline{\varphi_{c}}}
\end{aligned}
$$

Through the above analysis, and then calculate the probability of damage. Probabilistic calculations are based on the assumption that the damage caused by personal injury and breakage is not taken into account; the damage caused by the secondary effect is not considered; in the case of combat mode and condition known, the position of the missile hit the radar radiation source is randomly distributed.

When the ARM Warhead explodes, it is assumed that the damage to any of the two critical parts of the radiation source is an independent event that can be considered as a non-destructive accumulation condition. According to the principle of probability, it can be seen that damage to any critical component under the condition of no damage accumulation will damage the whole radar radiation source. The radiation damage pattern of radar radiation source is as follows:

$$
G(X, Y, Z)=1-\prod_{i=1}^{K}\left[1-p_{i}(X, Y, Z)\right]
$$

$p_{i}(X, Y, Z)$ is the probability that the warhead will damage the $\mathrm{i}$-th piece when it explodes at point $(X, Y, Z) ; \mathrm{K}$ is the number of vital parts. Assuming that $p(m)$ is the probability of hitting the block fragments on the critical components of the radar radiation source, $\mathrm{A}$ is the probability of damage to the $\mathrm{m}$ pieces broken on the part. By the full probability formula available:

$$
p_{i}(X, Y, Z)=\sum_{m=0}^{\infty} p(m) \cdot G(m)
$$

Law of the fragment by normal distribution description:

$$
\Psi_{R}\left(\varphi_{R}\right)=\frac{1}{\sqrt{2 \pi} \sigma_{\varphi_{R}}} \exp \left[-\frac{\left(\varphi_{R}-\overline{\varphi_{R}}\right)^{2}}{2 \sigma_{\varphi_{R}}^{2}}\right]
$$

Among them, $\Psi_{R}\left(\varphi_{R}\right)$ is the relative scattering average distribution density and $\varphi_{R}$ is the mathematical expectation of relative motion scattering. 


$$
\sigma\left(\varphi_{R}\right)=\frac{1}{(2 \pi)^{3 / 2} \sigma_{\varphi_{R}} \sin \varphi_{R}} \exp \left[-\frac{\left(\varphi_{R}-\overline{\varphi_{R}}\right)^{2}}{2 \sigma_{\varphi_{R}}^{2}}\right]
$$

The number of fragments between the critical parts of the radar radiation source:

$$
\bar{m}=\frac{N S_{O T} \sin ^{2}\left(\overline{\varphi_{p}}-\gamma \cos \beta\right)}{(2 \pi)^{3 / 2} \rho^{2} \sigma_{\varphi_{R}} \sin \varphi_{p}} \exp \left[-\frac{\left(\varphi_{p}-\overline{\varphi_{R}}\right)^{2}}{2 \sigma_{\varphi_{R}}^{2}}\right]
$$

Among them, $S_{O T}$ is the key parts of the area, $\rho, \beta, \varphi_{p}$ is the key parts of the center in the bombing point as the origin of the spherical coordinates of the ball, $\gamma$ for the radar radiation source - fragments of the relative coordinate system and the rotation angle between the body coordinate system [5].

Assume that a ARM fragment warhead, the number is 2000 , the static flying speed is $1020 \mathrm{~m} / \mathrm{s}$, the relative motion of flying fragments is expected to 60 degrees, when the warhead explosion, if a crucial part of the projection area of 0.5 square meters, as shown in Figure 2 for the number of the fragments and hit fall curve distance.

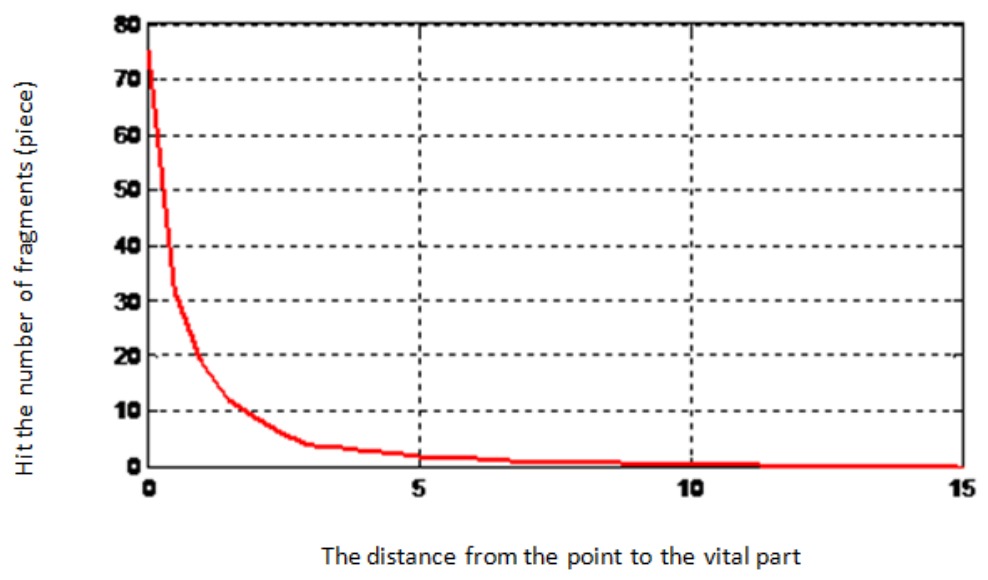

Fig. 2 the relationship between the number of hits and the landing point distance

For the fragmented warhead, the total number of fragments $\mathrm{N}$ is much larger than the number $\mathrm{m}$ of fragments that hit a critical component of the radar radiation source, which is in accordance with the Poisson distribution conditions:

$$
p_{N}(k=m)=C_{N}^{m} p^{m} q^{N-m} \approx \frac{\lambda^{m}}{m !} e^{-\lambda}
$$

Where $\lambda=n p=\bar{m}, \bar{m}$ is expected to hit the vital parts of the fragment count. For the condition of no damage accumulation, the damage probability of the coordinates is subject to the law of exponential damage:

$$
G(m)=1-\left(1-p_{1}\right)^{m}
$$

Among them, the probability that the key component is damaged for a fragment. Integrated formula(13) to formula(19), we can get:

$$
G(X, Y, Z)=1-\exp \left[-\sum_{i=1}^{K} \bar{m}_{i}(X, Y, Z) p_{1 i}\right]
$$

Among them, $p_{1 i}$ is the i-th critical components of a single fragment damage probability [6].

\section{Conclusion}

It is very important to improve the hit accuracy of ARM by studying the effective damage probability of anti radiation missile. By calculating the damage probability, we can judge the performance of ARM against active decoy. In this paper, the effect evaluation model is deeply studied, 
and the probability of damage and the probability of anti decoy are given, which makes the evaluation more accurate, reliable and scientific.

\section{References}

[1]. Mingshun Ai: Research on signal processing technology of ARM against active decoy, Ph.D., China, 2011.

[2]. Fuzhong Bai. Active decoy system against ARM scheme. Winged missile. 2011.

[3]. Yuanjun Ma. Radar active decoy design. Radar and countermeasure. Vol.2002(2), p. 32-35.

[4]. Yankui Wang. Analysis of ARM seeker technology development. Winged missile.Vol.2009(3), p. 39-44.

[5]. Hongsheng Li. Analysis of ARM induced polarization performance. Fire Control Radar Technology. Vol. 2006, 35(3), p. 42-44.

[6]. Mingshun Ai, Hongguang Ma. An evaluation index of ARM anti active decoy performance. Vol. 2010, 32(10), p. 13-16. 